\section{Modifying factors related to asymmetric diabetic retinopathy}

MURAT DOGRU, MASANORI INO-UE, MAKOTO NAKAMURA, MISAO YAMAMOTO

\begin{abstract}
Purpose To identify the modifying factors related to the development of proliferative diabetic retinopathy (PDR).

Methods Thirty-eight eyes of 19 non-insulindependent diabetes mellitus (NIDDM) patients with maintained asymmetric PDR were retrospectively reviewed.

Results Five patients with ipsilateral carotid stenoses $\geqslant 90 \%$ had PDR. Four patients with high myopia over 6 dioptres and 4 patients with optic atrophy and at least a quadrant defect in the visual field had non-proliferative diabetic retinopathy. Of 6 patients with unilateral asteroid hyalosis, 5 had no posterior vitreous detachment (PVD) and PDR.

Conclusion Two factors reached statistical significance as factors modifying PDR: carotid occlusive disease and PVD. Optic atrophy and high myopia showed trends of being a protective influence.
\end{abstract}

Key words Proliferative diabetic retinopathy, Non-insulin-dependent diabetes mellitus

Diabetes mellitus is a systemic disease in which retinopathy usually develops in a symmetric pattern over a long period of time. With respect to the ocular complications of diabetes, asymmetric diabetic retinopathy is considered the exception rather than the rule. Previous investigators have suggested several local and systemic factors to account for the development of asymmetric diabetic retinopathy. ${ }^{1-3}$

Identification of such factors may be important in planning appropriate treatment strategies. Moreover, asymmetry involving proliferative diabetic retinopathy (PDR) or maculopathy is very important clinically, because severe visual loss often accompanies this type of retinopathy. We retrospectively reviewed a series of patients with maintained asymmetric retinopathy to identify some of the risk and protective factors for the development of PDR.

\section{Materials and methods}

Early Treatment of Diabetic Retinopathy Study (ETDRS) criteria were used to define various stages of diabetic retinopathy. ${ }^{4,5}$ Asymmetric retinopathy was defined as PDR in one eye and non-proliferative diabetic retinopathy (NPDR) or no retinopathy in the fellow eye persisting for more than 2 years. Thirty-eight eyes of 19 patients examined at Kobe University School of Medicine, Department of Ophthalmology, Diabetes Outpatient Clinic during 1991-1998 who met this requirement were recruited. A concurrent systemic diagnosis of diabetes mellitus and presence of data about the state of diabetes control were also required for inclusion. Any patient with a secondary systemic illness associated with proliferative retinopathy, such as sickle cell anaemia or sarcoidosis, was excluded from the study. All patients had intravenous fluorescein angiograms available, the evaluation of which was carried out by the same clinician. The diagnosis of PDR was made when new vessels were observed in the posterior segment. We sought only patients with 'high-risk' characteristics in one eye and neither proliferative nor preproliferative disease in the fellow eye, in order to eliminate those patients whose retinopathy was only mildly asymmetric. Patients who received laser treatment before the first identification of asymmetric retinopathy were excluded. If patients received any laser treatment to the eye with NPDR during the follow-up period, they were also excluded.

None of the eyes in this study had a history of cataract or retinal surgery and intraocular disease that might have influenced the development of PDR. Patients with delayed arm-retina circulation time on fluorescein angiography also underwent ophthalmodynamometry and were referred to the Department of Internal Medicine for angiography and Doppler ultrasonography to rule out carotid occlusive disease. Patients who were confirmed as having carotid stenoses were then referred to our department for further follow-up. Goldmann visual field examination records were available for patients with optic atrophy (OA). Examinations of vitreous status in patients for whom data about posterior vitreous detachment (PVD) were available had been performed using a slit-lamp and an aspheric +60 dioptre preset lens. The condition of the vitreous was recorded according to the classification of Takahashi and associates. ${ }^{6}$ Intraocular

\author{
M. Dogru \\ M. Ino-ue \\ M. Nakamura \\ M. Yamamoto \\ Kobe University School of \\ Medicine \\ Department of \\ Ophthalmology \\ Kobe, Japan
}

Masanori Ino-ue, MD, PhD Kobe University School of

Medicine

Department of

Ophthalmology

650-0017 Kobe Chuo-ku

Kusunoki-cho 7-5-2

Japan

Tel: +81783417451 ext 5682

Fax: +81 783613154

e-mail mymam

956@mailgate.kobe-u.ac.jp 
Table 1. Asymmetric diabetic retinopathy and significant carotid artery disease

\begin{tabular}{ccccc}
\hline & & & \multicolumn{2}{c}{ Diabetic retinopathy } \\
\cline { 3 - 4 } Sex/Age (years) & Diabetes duration (years) & Carotid stenosis (\%) & OD & OS \\
\hline M/59 & 10 & L: $90 \%$ & NPDR & PDR (INV) \\
M 61 & 17 & L: $100 \%$ & NPDR & PDR (INV) \\
M 62 & 15 & L: $100 \%$ & PDR & NPDR \\
M 65 & 16 & R: $99 \%$ & NPDR & PDR (INV) \\
M $/ 59$ & 13 & L: $100 \%$ &
\end{tabular}

L, left; $R$, right; OD, right eye; OS, left eye; NPDR, non-proliferative diabetic retinopathy; PDR, proliferative diabetic retinopathy; INV, iris neovascularisation.

characteristics that were thought to have an influence on asymmetric retinopathy were then analysed using Statview software (1988; Abacus Concepts, CA).

The analysis of categorised data was carried out by the chi-squared test, with the probability level for statistical significance set at $5 \%$.

\section{Results}

We encountered asymmetric diabetic retinopathy in 5 patients with ipsilateral carotid stenoses, 4 with OA, 4 with unilateral high myopia and 6 with asteroid hyalosis $(\mathrm{AH})$. All the patients in this study had well-controlled NIDDM. The average age was $61 \pm 9$ years and the average duration of diabetes was $12 \pm 4$ years. There were 19 eyes with PDR, of which 8 had early and 11 had high-risk proliferative retinopathy. Nine mild and 9 moderate cases of NPDR were encountered in the fellow eyes. Only one eye with high myopia did not have diabetic retinopathy.

Five eyes with delayed arm-retina circulation time on fluorescein angiography also had low retinal artery perfusion pressure on ophthalmodynamomety. These 5 eyes with PDR were confirmed to have ipsilateral carotid stenoses $\geqslant 90 \%$ after carotid angiography and/or Doppler ultrasonography (Table 1). The fellow eyes had NPDR. Three eyes with $100 \%$ carotid stenoses also had iris neovascularisation (INV).

Four eyes had OA that was due to anterior ischaemic optic neuropathy (AION) in 2 eyes and mild blunt trauma in one eye. The aetiology of OA in one eye was unknown. Goldmann perimetry showed contraction of the visual field by one quadrant or more in these eyes, as shown in Table 2. All eyes with OA had NPDR.

Table 2. Asymmetric diabetic retinopathy and optic atrophy

\begin{tabular}{|c|c|c|c|c|c|}
\hline \multirow{2}{*}{$\begin{array}{l}\text { Sex / Age } \\
\text { (years) }\end{array}$} & \multirow{2}{*}{$\begin{array}{c}\text { Diabetes duration } \\
\text { (years) }\end{array}$} & \multirow[b]{2}{*}{ Cause of $\mathrm{OA}$} & \multirow[b]{2}{*}{ Visual field } & \multicolumn{2}{|c|}{ Diabetic retinopathy } \\
\hline & & & & OD & OS \\
\hline$M / 58$ & 10 & $\mathrm{R}$ : Blunt trauma & & NPDR & PDR \\
\hline $\mathrm{M} / 43$ & 15 & L: AION & & PDR & NPDR \\
\hline $\mathrm{M} / 47$ & 19 & R: AION & & NPDR & PDR \\
\hline $\mathrm{M} / 77$ & 7 & L: Unknown & & PDR & NPDR \\
\hline
\end{tabular}

L, left; R, right; OD, right eye; OS, left eye; OA, optic atrophy; AION, anterior ischaemic optic neuropathy; PDR, proliferative diabetic retinopathy; NPDR, non-proliferative diabetic retinopathy. 


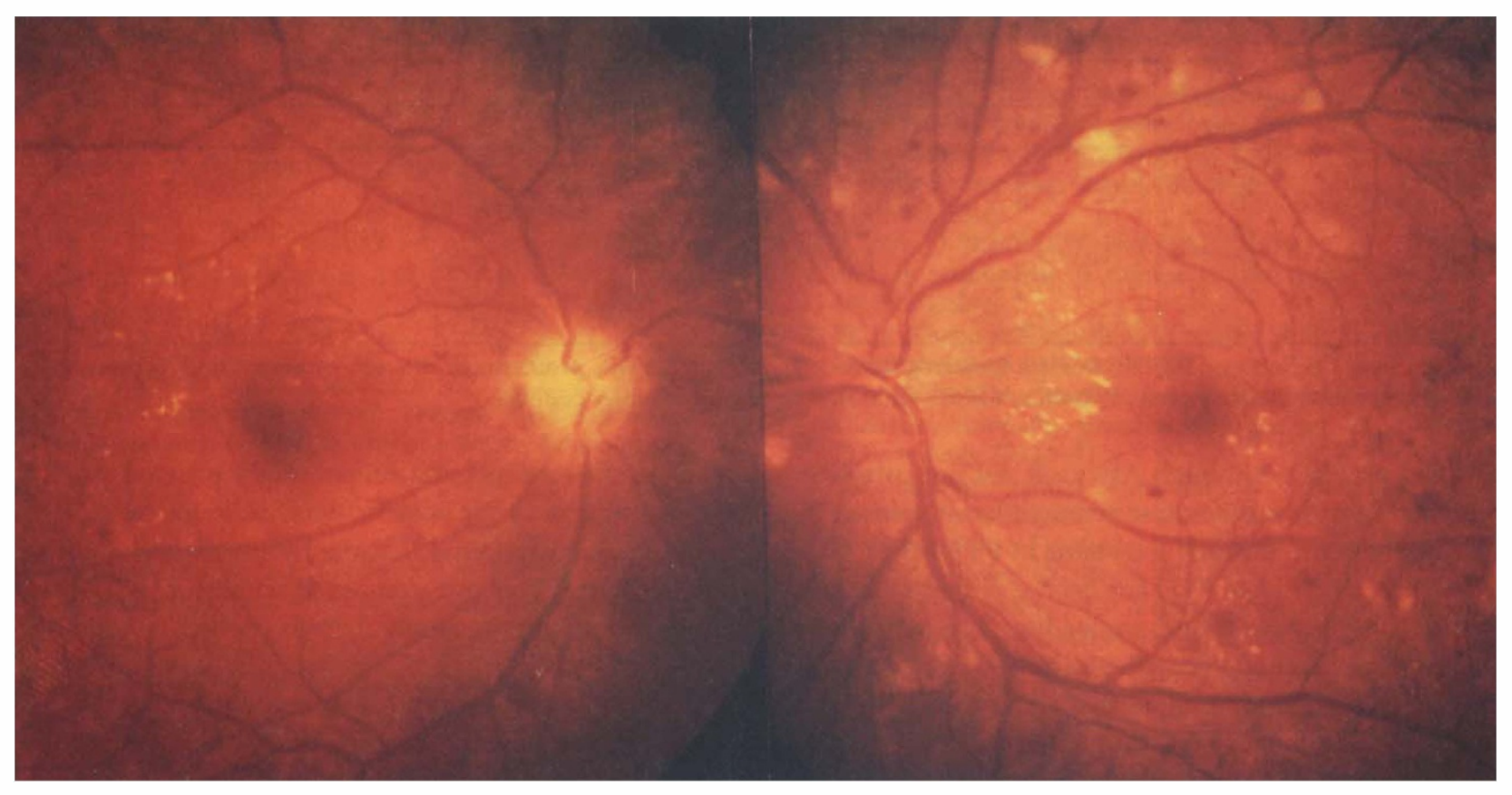

Fig. 1. Patient with asymmetric diabetic retinopathy and optic atrophy. There is non-proliferative diabetic retinopathy in the eye with optic atrophy, and soft exudates, dot-blot, nerve fibre layer haemorrhages and retinal neovascularisations in the fellow eye.

Asymmetric retinopathy in a patient with OA is shown in Fig. 1. Only 4 eyes had significant anisometropia with high myopia. These eyes had either no diabetic retinopathy or NPDR and all had complete PVD (Table 3). The remaining eyes in the study were within 1 dioptre of emmetropia and the interocular refractive difference did not exceed 2 dioptres. Unilateral $\mathrm{AH}$ was encountered in 6 eyes, 5 of which had no PVD. Only one eye had complete PVD, as shown in Table 4. All eyes with AH but without PVD had PDR. Three fellow eyes without AH had complete PVD and NPDR. One fellow eye had partial PVD and PDR, whereas 2 other eyes without PVD showed NPDR. The relation between PDR and the absence of PVD was significant $(p=0.03)$. A patient with $\mathrm{AH}$ and asymmetric diabetic retinopathy is shown in Fig. 2.

No PVD was described in 2 eyes with NPDR and 10 eyes with PDR. Absence of PVD and ipsilateral carotid occlusion showed an influence on the development of PDR and reached statistical significance, whereas complete PVD emerged as a significant protective factor against PDR. None of the other factors showed a statistically significant imbalance between eyes with PDR, NPDR or no diabetic retinopathy, although certain trends were suggested. Optic atrophy and high myopia showed trends of being a protective influence. $\mathrm{AH}$ itself did not relate to PDR significantly (Table 5).

\section{Discussion}

Asymmetric retinopathy occurs in $5-10 \%$ of diabetic patients with PDR. ${ }^{2-7}$ Various factors such as vitreous loss at cataract surgery, trauma, uveitis, optic atrophy, ipsilateral carotid obstructive disease, branch retinal vein occlusion, PVD, chorioretinal atrophy, amblyopia and high myopia have been suggested to induce asymmetric diabetic retinopathy. ${ }^{13,8,9}$ The natural course and outcome of diabetic retinopathy may be influenced by such factors. In this series, two local factors - ipsilateral carotid occlusive disease and PVD - were shown as significant modifiers of PDR.

Complete PVD is accepted to be associated with NPDR or with mild degrees of proliferation whereas vitreous traction is a grave prognostic sign indicating a high risk that PDR will progress. Eyes with partial PVD are at risk of inducing vitreous traction at sites of new vessel formation. ${ }^{10-13}$ In this study we evaluated the relation between PVD and PDR in eyes with AH. Interestingly, we observed that $83 \%$ of the $\mathrm{AH}$ eyes with

Table 3. Asymmetric diabetic retinopathy and refractive error

\begin{tabular}{|c|c|c|c|c|c|c|c|}
\hline \multirow{2}{*}{$\begin{array}{l}\text { Sex/Age } \\
\text { (years) }\end{array}$} & \multirow{2}{*}{$\begin{array}{c}\text { Diabetes duration } \\
\text { (years) }\end{array}$} & \multicolumn{2}{|c|}{ PVD } & \multicolumn{2}{|c|}{ Refractive error (dioptres) } & \multicolumn{2}{|c|}{ Diabetic retinopathy } \\
\hline & & OD & OS & OD & OS & OD & OS \\
\hline $\mathrm{M} / 63$ & 11 & C & No & -12 & +1 & NPDR & PDR \\
\hline $\mathrm{M} / 62$ & 10 & No & C & -1 & -6 & PDR & NPDR \\
\hline $\mathrm{F} / 43$ & 9 & C & No & -10 & -1 & NPDR & PDR \\
\hline $\mathrm{M} / 70$ & 10 & C & No & -10 & -1.5 & NPDR & PDR \\
\hline
\end{tabular}

OD, right eye; OS, left eye; PDR, proliferative diabetic retinopathy; NPDR, non-proliferative diabetic retinopathy; PVD, posterior vitreous detachment, C, complete. 
Table 4. Asymmetric diabetic retinopathy and asteroid hyalosis

\begin{tabular}{|c|c|c|c|c|c|c|c|}
\hline \multirow{2}{*}{$\begin{array}{l}\text { Sex/Age } \\
\text { (years) }\end{array}$} & \multirow{2}{*}{$\begin{array}{c}\text { Diabetes duration } \\
\text { (years) }\end{array}$} & \multicolumn{2}{|c|}{ Asteroid hyalosis } & \multicolumn{2}{|c|}{ PVD } & \multicolumn{2}{|c|}{ Diabetic retinopathy } \\
\hline & & OD & OS & OD & OS & OD & OS \\
\hline $\mathrm{F} / 68$ & 17 & + & - & No & No & PDR & NPDR \\
\hline$M / 63$ & 6 & + & - & No & $\mathrm{C}$ & PDR & NPDR \\
\hline $\mathrm{M} / 70$ & 12 & - & + & No & No & NPDR & PDR \\
\hline $\mathrm{M} / 70$ & 15 & - & + & $\mathrm{P}$ & C & PDR & NPDR \\
\hline$M / 69$ & 17 & + & - & No & $\mathrm{C}$ & PDR & NPDR \\
\hline$M / 59$ & 5 & + & - & No & C & PDR (CSME) & NPDR \\
\hline
\end{tabular}

OD, right eye; OS, left eye; NPDR, non-proliferative diabetic retinopathy; PDR, proliferative diabetic retinopathy; PVD, posterior vitreous detachment; $\mathrm{C}$, complete; $\mathrm{P}$, partial; CSME, clinically significant macular oedema.

PDR had no PVD. Although AH itself did not correlate significantly with proliferative retinopathy, it seemed to exert an influence through the status of PVD. The prevalence of complete PVD has been reported to be low in patients with $\mathrm{AH} .{ }^{14-17}$ It has also been reported that partial PVD is rarely noted in healthy eyes, since the PVD process is believed to be rapid and completed within a few hours after its incidence. ${ }^{12}$ Partial or no PVD may be a more frequent finding in eyes with asteroid bodies than in healthy eyes because the vitreous body may be less elastic and thus less able to detach from the more adherent sites of vitreoretinal adhesion such as the optic disc or the macula. The low incidence of PVD in these eyes may prove to have some relation with the failure of the vitreous to contract completely in the presence of asteroid bodies. In this study, all eyes with $\mathrm{AH}$ but without PVD had PDR. Complete PVD in 3 fellow eyes without $\mathrm{AH}$ was associated with NPDR and might have acted as a protective factor against PDR. However, there were 2 fellow eyes without PVD and AH that did not develop PDR. Therefore, we believe that if PVD has not taken place before the initiation of ischaemic retinal changes, vitreoretinal attachment might provide the necessary support for neovascularisation. Indeed, a histological study found that the fibrous material of the vitreous cortex was interconnected with and incorporated into the newly formed proliferative tissue in PDR. ${ }^{18}$

Another important factor that related to PDR was carotid stenosis. Moderate ipsilateral carotid obstructive disease has previously been shown to be associated with protection against PDR. The protective effect of ipsilateral carotid occlusive disease has been speculated to be a result of reduction in the retinal arterial perfusion pressure. ${ }^{19,20}$ However, Duker et al. ${ }^{7}$ contradicted this hypothesis and reported that two of their patients had PDR ipsilateral to severe carotid stenoses. The current study found 5 patients with haemodynamically significant ipsilateral carotid lesions, all of whom had $\geqslant 90 \%$ carotid stenoses and PDR on the involved side. Three of these patients also had rubeosis iridis. These patients had the additive effects of both the ocular ischaemic syndrome and diabetes to account for their neovascularisation. Our findings suggest that carotid
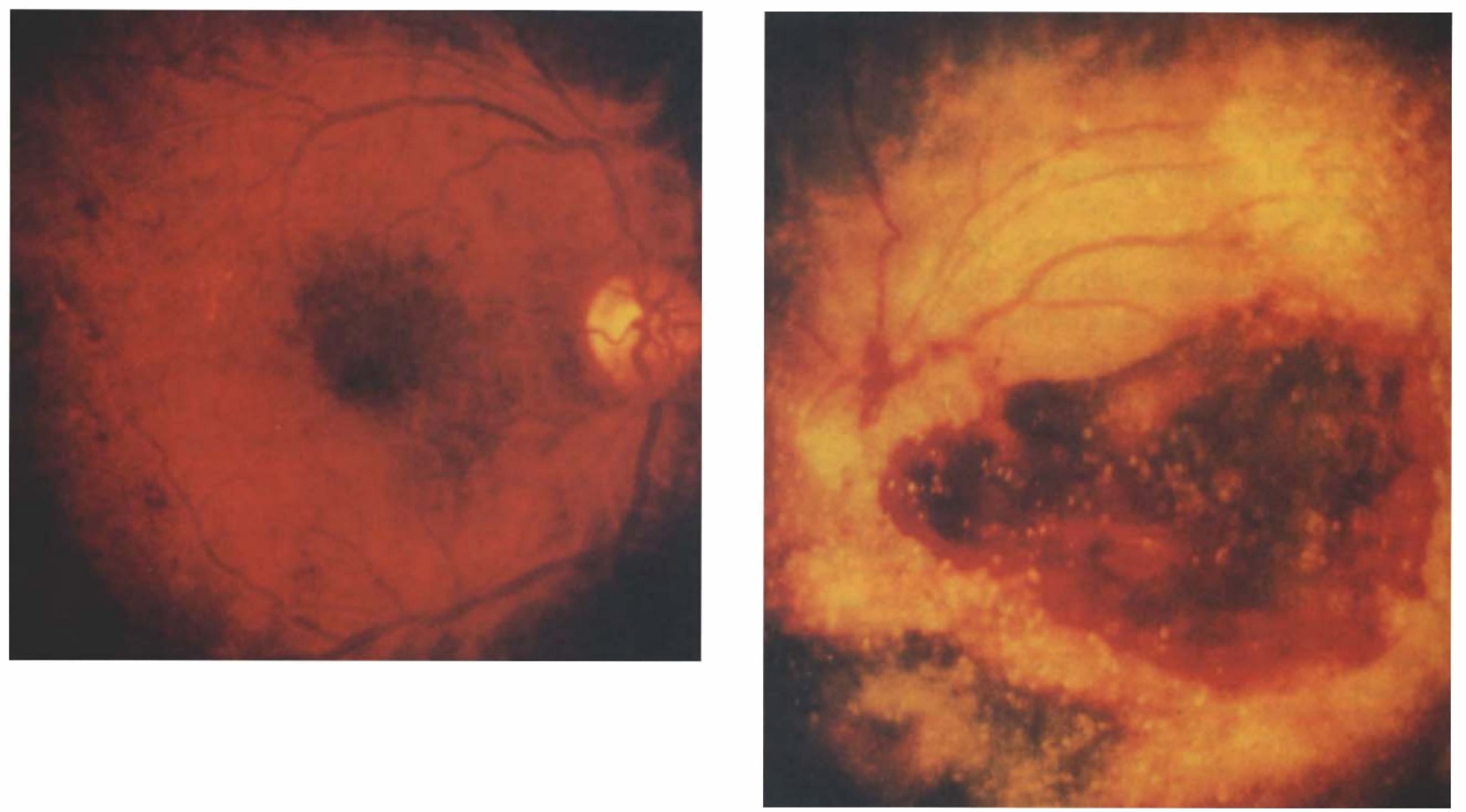

Fig. 2. Patient with asymmetric diabetic retinopathy and asteroid hyalosis. There is proliferative diabetic retinopathy with preretinal haemorrhage in the eye with asteroid hyalosis and non-proliferative diabetic retinopathy in the fellow eye. 
Table 5. Ocular modifying factors between eyes with non-proliferative and proliferative diabetic retinopathy

\begin{tabular}{lcrc}
\hline Study factors & NPDR & PDR & $\begin{array}{c}\text { Fisher's exact } \\
p \text { value }\end{array}$ \\
\hline PVD & & & \\
$\quad$ Complete & 8 & 0 & 0.001 \\
$\quad$ No & 2 & 10 & 0.023 \\
Ipsilateral carotid stenosis & 0 & 5 & 0.023 \\
Optic atrophy & 4 & 0 & 0.052 \\
High myopia & 4 & 0 & 0.052 \\
Asteroid hyalosis & 1 & 5 & 0.099 \\
\hline
\end{tabular}

NPDR, non-proliferative diabetic retinopathy; PDR, proliferative diabetic retinopathy; PVD, posterior vitreous detachment.

occlusion exceeding $90 \%$ aggravates diabetic retinopathy. High myopia and optic atrophy showed trends towards being a protective influence. A larger study might have demonstrated statistical significance of these factors that failed to reach significance in our study. All 4 cases with high myopia in this series had complete PVD and NPDR. It is suggested that myopia of 5 dioptres or more protects against PDR. Speculation as to the mechanism for the relationship between myopia and retinopathy involves the elongation of the eye with deformation and atrophy of the posterior pole leading to decreased retinal metabolism, alteration of the ocular blood flow and very frequent association with complete PVD. ${ }^{3,21,22}$ In addition, retinochoroidal degeneration may elaborate transforming growth factor beta (TGF $\beta$ ), which is a cytokine protecting against proliferation by a mechanism similar to that seen in retinal photocoagulation. ${ }^{23}$ All these factors may act as protective influences alone or in combination.

The protective effect of OA against PDR and its mechanism is still under debate. The loss of retinal nerve fibres along with the atrophic process may reduce the metabolic requirements in such eyes and secondarily reduce the ischaemic stimulus for proliferation., ${ }^{8,24} \mathrm{~A}$ decrease in blood flow in the retinal vessels and a reduction in oxygen saturation have been shown in eyes with OA. ${ }^{25}$ In this study, eyes with OA had NPDR and visual field defects extending over a quadrant of the field were seen to be associated with a protective effect against PDR. However, the visual field defects did not correspond to areas of asymmetric retinopathy, and how the nerve fibre damage relates to protection from retinopathy has yet to be clarified through further qualitative and quantitative studies.

In conclusion, PVD and significant ipsilateral carotid occlusive disease are risk factors for PDR. Complete PVD, optic atrophy and high myopia protect against the progression of PDR. Our report may serve as a guide in patients with asymmetric PDR and may influence treatment strategies. Further investigation in identifying unknown intraocular factors accounting for asymmetric retinopathy may provide more information about incidence and progression of PDR.

\section{References}

1. Ulbig MRW, Hamilton AMP. Factors influencing the natural course of diabetic retinopathy. Eye 1993;7:242-9.

2. Valone AJ, McMeel W, Franks EP. Unilateral proliferative diabetic retinopathy. I. Initial findings. Arch Ophthalmol 1981;99:1357-61.

3. Valone AJ, McMeel W, Franks EP. Unilateral proliferative diabetic retinopathy. II. Clinical course. Arch Ophthalmol 1981;99:1362-6.

4. Early Treatment Diabetic Retinopathy Study Group. Ophthalmology 1991;98:739-834.

5. Olk RJ, Lee CM. Classification of diabetic retinopathy. In: Diabetic retinopathy: practical management. JB Lippincott, Philadelphia, 1993:3-21.

6. Takahashi M, Trempe CL, Maquire K. Vitreoretinal relationship in diabetic retinopathy: a biomicroscopic evaluation. Arch Ophthalmol 1981;99:241-5.

7. Duker JS, Brown GC, Bosley TM, Colt CA, Reber R. Asymmetric proliferative diabetic retinopathy and carotid artery disease. Ophthalmology 1990;90:869-74.

8. Browning DJ, Flynn HW, Blankenship GW. Asymmetric retinopathy in patients with diabetes mellitus. Am J Ophthalmol 1988;105:584-9.

9. Moss SE, Klein R, Klein BEK. Ocular factors in the incidence and progression of diabetic retinopathy. Ophthalmology 1994;101:77-83.

10. Akiba J, Arzabe CW, Trempe CL. Posterior vitreous detachment and neovascularisation in diabetic retinopathy. Ophthalmology 1990;97:889-91.

11. Davis MD. Vitreous contraction in proliferative diabetic retinopathy. Arch Ophthalmol 1965;74:741-51.

12. Jalkh A, Takahashi M, Topilow HW, Trempe CL, McMeel W. Prognostic value of vitreous findings in diabetic retinopathy. Arch Ophthalmol 1982;100:432-4.

13. Tolentino FL, Lee PF, Schepens CL. Biomicroscopic study of vitreous cavity in diabetic retinopathy. Arch Ophthalmol 1966;75:238-46.

14. Bergren RL, Brown GC, Duker JS. Prevalence and association of asteroid hyalosis with systemic diseases. Am J Ophthalmol 1991;111:289-93.

15. Kojima K, Ishigura H, Harada T, Harada K, Awaya S. Clinical and histopathological study of asteroid hyalosis in diabetics. Jpn J Clin Ophthalmol 1987;41:579-83.

16. Topilow HW, Kenyon KR, Takahashi M. Biomicroscopy, ultrastructure and composition. Arch Ophthalmol 1982;100:964-8.

17. Wasano T, Hirokawa H, Tagawa H, Trempe CL, Buaney SM. Asteroid hyalosis: posterior vitreous detachment and diabetic retinopathy. Ann Ophthalmol 1987;19:255-8.

18. Faulborn J, Bowald S. Microproliferations in proliferative diabetic retinopathy and their relationship to the vitreous: corresponding light and electronmicroscopic studies. Graefes Arch Clin Exp Ophthalmol 1985;223:130-8.

19. Brown GC, Magargal LE, Simeone FA, Goldberg RE, Federman JL, Benson WE. Arterial obstruction and ocular neovascularization. Ophthalmology 1982;89:139-44.

20. Gay AJ, Rosenbaum AL. Retinal artery pressure in asymmetric diabetic retinopathy. Arch Ophthalmol 1966;75:758-62.

21. Avetisov ES, Savitskaya NF. Some features of ocular microcirculation in myopia. Ann Ophthalmol 1977;9:1261-4.

22. Jain IS, Luthra CL, Das T. Diabetic retinopathy and its relation to errors of refraction. Arch Ophthalmol 1967;77:59-65.

23. D'Amore PA. Mechanisms of retinal and choroidal neovascularization. Invest Ophthalmol Vis Sci 1994;35:3974-9.

24. Weiter JJ, Zuckerman R. The influence of the photoreceptorRPE complex on the inner retina: an explanation for the beneficial effects of photocoagulation. Ophthalmology 1980;87:1133-41.

25. Sebag J, Delori FC. Effects of optic atrophy on retinal blood flow and oxygen saturation in humans. Arch Ophthalmol 1989;107:222-6. 